\title{
Waveguided spoof surface plasmons with deep-subwavelength lateral confinement
}

\author{
Diego Martin-Cano, ${ }^{1}$ O. Quevedo-Teruel, ${ }^{1}$ Esteban Moreno, ${ }^{1}$ L. Martin-Moreno, ${ }^{2}$ and F. J. Garcia-Vidal ${ }^{1, *}$ \\ ${ }^{1}$ Departamento de Fisica Teorica de la Materia Condensada, Universidad Autonoma de Madrid, E-28049 Madrid, Spain \\ ${ }^{2}$ Instituto de Ciencia de Materiales de Aragon (ICMA) and Departamento de Fisica de la Materia Condensada, \\ CSIC-Universidad de Zaragoza, E-50009 Zaragoza, Spain \\ ${ }^{*}$ Corresponding author: fj.garcia@uam.es
}

Received August 11, 2011; revised October 24, 2011; accepted October 24, 2011;

posted October 26, 2011 (Doc. ID 152749); published November 28, 2011

\begin{abstract}
We present a new type of waveguide scheme for terahertz circuitry based on the concept of spoof surface plasmons. This structure is composed of a one-dimensional array of $L$-shaped metallic elements horizontally attached to a metal surface. The dispersion relation of the surface electromagnetic modes supported by this system presents a very weak dependence with the lateral dimension and the modes are very deep-subwavelength confined with a long-enough propagation length. (C) 2011 Optical Society of America

OCIS codes: $240.6680,230.7370,230.7390,240.6690,170.4520,050.6624$.
\end{abstract}

The terahertz (THz) electromagnetic (EM) spectrum covers one of the most interesting and multidisciplinary research fields in science nowadays [1,2] due to its potential applications in astronomy [3], medicine [4] , imaging [5], and information processing [6]. An important goal within the THz research is the development and integration of photonic circuits that must be efficiently coupled to THz sources and detectors [7]. Within this endeavor, several approaches for THz waveguides (TWGs) have been proposed recently, mainly divided in dielectric [8] and metallic TWGs $[9,10]$. In general, the miniaturization of dielectric waveguides is diffraction-limited and they are not monolithic, which make their integration more difficult for circuitry purposes. Within the metallic approach, the concept of spoof surface plasmons [11, 12] when applied to the design of TWGs has proven to be a very promising route [13-19].

In this Letter we present a new design for a TWG, based on the concept of spoof surface plasmons. The structure consists of a periodic chain of $3 \mathrm{D} L$-shaped metallic particles attached to a metallic substrate. The surface EM modes supported by this system possess a very deep-subwavelength confinement around the waveguide and propagation lengths that are much larger than the wavelength. Importantly, the effective mode index of these spoof surface plasmons is very insensitive to the lateral dimension, which allows the design of energy concentration strategies in which the EM field transported by a broad TWG is focused into a very narrow one.

A sketch of the structure analyzed in this work is shown in the inset of Fig. 1. This illustration also displays the geometrical parameters of the TWG: the periodicity $d$, particle base-width $g$, interparticle spacing $a$, lateral width $L$, and the short and long sides of the letter $L, h$ and $h^{\prime}$, respectively. Two-dimensional arrays of mushroom-shaped metal particles (similar to $L$-shaped) have been studied before [20,21], mainly in the context of plasmonic metamaterial surfaces. Here we propose to reduce the dimensionality, aiming for one-dimensional (1D) $\mathrm{THz}$ waveguiding.

The dispersion relations displayed in Fig. 1 are obtained from a numerical solution of Maxwell's equations using a finite element method (COMSOL multiphysics).
These complex bands are solutions of the corresponding eigenvalue problem for a single unit cell, where Bloch boundary conditions are imposed in the propagation direction. In order to take into account the inherent ohmic losses in the metal, we use surface impedance boundary conditions [22] with the dielectric permittivity of aluminum taken from [23]. This approximation is justified in the $\mathrm{THz}$ regime due to the nanometer sized skin depths in real metals [24]. Figure 1 displays the bands of the fundamental surface EM modes for different lateral sizes $(L=0.5 d, 1 d, 2 d, 4 d, \infty)$ with $h=0.2 d, h^{\prime}=0.6 d$, $g=0.06 d$, and $a=0.4 d$. All the geometrical parameters are referred to the period of the array, $d$, that is chosen to be $d=400 \mu \mathrm{m}$, in order to have an operative wavelength within the THz regime. We would like to emphasize that this set of parameters has been chosen for proof-ofprinciple purposes and that other sets of parameters would yield similar results.

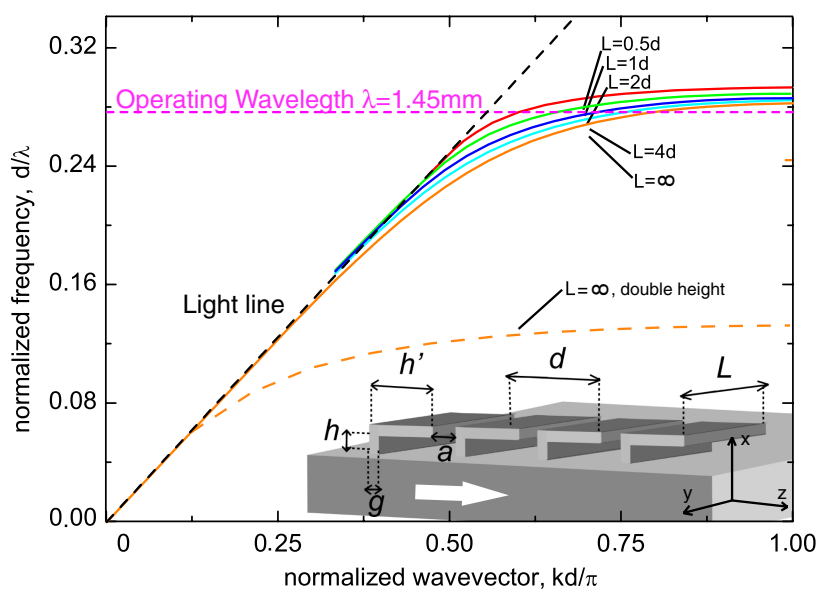

Fig. 1. (Color online) Inset: sketch of the structure under study with a description of the geometrical parameters (the arrow defines the mode propagation direction). Main panel: the bands associated with the fundamental EM modes supported by the system for $d=400 \mu \mathrm{m}, h=0.2 d, h^{\prime}=0.6 d$, $g=0.06 d$, and several lateral widths $L: L=0.5 d$ (red), $L=$ $1 d$ (green), $L=2 d$ (blue), $L=4 d$ (cyan), $L=\infty$ (orange). The band for the fundamental EM mode in which $h=d$ and $h^{\prime}=0.6 d$ and $L=\infty$ is also shown (dashed orange line). 
As observed in Fig. 1, the surface EM modes supported by the array of $L$-shaped metallic particles display dispersion relations resembling those of surface plasmon polaritons (SPPs) in the optical EM regime [25]. The bands lie outside the light cone, thus corresponding to confined propagating light modes, and reach an asymptote in a way very much similar to SPPs. The most remarkable property shown in Fig. 1 is the weak dependence of the bands with the lateral size $L$. It can be seen that relatively large lateral changes do not severely modify the bands. This small variation of the bands with lateral size has been previously predicted [16] and observed $[18,19]$ for the so-called domino structure. In the case of the domino SPPs, the asymptote frequency is mainly dictated by the height of the dominos [16]. Here, the sum of the two sides of the letter $L, h+h^{\prime}$, is playing a similar role. The empty space between consecutive metal particles seems to create an effective groove whose depth is defined by $h+h^{\prime}$ [26]. The resonance wavelength of this groove, $4\left(h+h^{\prime}\right)$, determines approximately the asymptote of the bands. From that estimation, we would obtain a normalized asymptotic wavelength, $d / \lambda$, of 0.31 , very close to that observed in Fig. 1, around $0.28 d$. Note that other geometrical factors like $\bar{g}$ and $a$ and the particular values of $h$ and $h^{\prime}$ also play a minor role in defining the asymptote frequency. Following that simple estimation, an increase by a factor of two on $h+h^{\prime}$ results in a reduction by half of the asymptote frequency, as observed in Fig. 1 (orange dashed line). The fact that the asymptote in the $\bar{L}$-shaped TWG can be tuned by two geometrical parameters $\left(h\right.$ and $\left.h^{\prime}\right)$ adds more flexibility to this TWG with respect to the domino one. Moreover, this TWG can also present a very high planar aspect ratio by making $h \ll h^{\prime}$, which could have important implications for fabrication purposes.

When evaluating the waveguiding capabilities of different surface EM modes propagating in metallic structures, a detailed study of the corresponding propagation lengths is mandatory. Figure 2(a) depicts the propagation length versus wavelength for the lateral sizes $L$ considered above. The propagation length (defined as the distance in which the incoming modal power has decreased to $1 / e$ of its initial value) is the inverse of the imaginary part of the complex wavevector, $l=1 /(2 \operatorname{Im} k)$, which can be extracted from an eigenvalue problem [25]. In general, it can be observed how the propagation length decreases at smaller wavelengths for all the lateral sizes. This is a consequence of the deeper confinement at higher frequencies due to the larger modal wavevectors (see Fig. 1). Nevertheless, there is a broad wavelength bandwidth presenting long-enough propagation lengths. For an operating wavelength of $1.45 \mathrm{~mm}$, the propagation length ranges from $8 \lambda$ (for $L=\infty$ ) to $24 \lambda$ (for $L=0.5 d$ ). The propagation length diminishes for larger lateral sizes at a fixed wavelength, which is also a consequence of its deeper confinement (see below).

Another important ingredient for the design of a compact $\mathrm{THz}$ circuitry is to evaluate the confinement of the surface EM modes. As can be seen in the upper and lower insets of Fig. 2(a), the electric and magnetic fields of the fundamental surface EM mode are very strongly confined to the waveguide. In order to evaluate this localization more quantitatively, we make use of the modal size $\delta$,
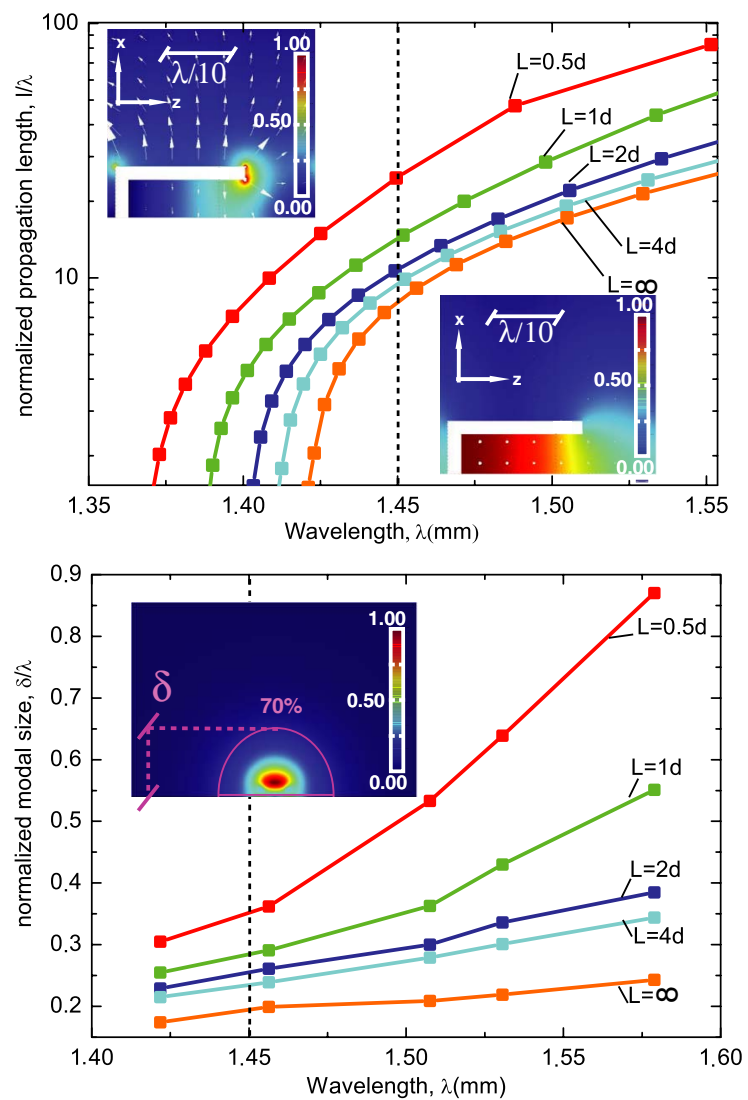

Fig. 2. (Color online) (a) Normalized propagation length of the TWG under study for different $L s$ as a function of $\lambda$. Upper (lower) inset: the electric (magnetic) field contour cut along a $x y$-plane that shows a single unit cell for $L=\infty$ and evaluated at $\lambda=1.45 \mathrm{~mm}$. (b) Normalized modal size $\delta$ (see definition in the main text) for different $L$ s as a function of $\lambda$. Inset: Poynting vector field (modulus) distribution in a transverse $x y$-plane placed between the particles also evaluated at $\lambda=1.45 \mathrm{~mm}$ for $L=0.5 d$. The magenta line shows the area that carries $70 \%$ of the total modal power. In both panels, the geometrical parameters are the same as those used in Fig. 1 .

defined as the transverse size corresponding to the circular area that carries $70 \%$ of the modal power (see the inset of Fig. 2(b) for an illustration). Figure 2(b) shows the evolution of $\delta$ as a function of the wavelength for the lateral sizes considered above. As expected, the modal size diminishes at higher frequencies until the asymptote frequency is reached. Notice the very deep-subwavelength confinement associated with the surface EM modes: at the operating wavelength $\lambda=1.45 \mathrm{~mm}$, the modal size ranges from $0.19 \lambda$ (for $L=\infty$ ) to $0.36 \lambda$ (for $L=0.5 d$ ). As it occurs for SPPs in the optical regime [17], there is an operational trade-off between confinement and propagation losses, i.e., longer propagation lengths imply larger modal sizes and vice versa.

The two main characteristics of the surface EM modes supported by a periodic array of $L$-shaped metallic particles, namely, insensitivity to $L$ and long-enough propagation length, can be exploited to concentrate $\mathrm{THz}$ energy along the waveguide, by using, for instance, a tapered configuration (such as in Fig. 3). In this structure, the lateral width of the waveguide is reduced in the direction of propagation. As the effective mode propagation constant presents a weak dependence with lateral width, 


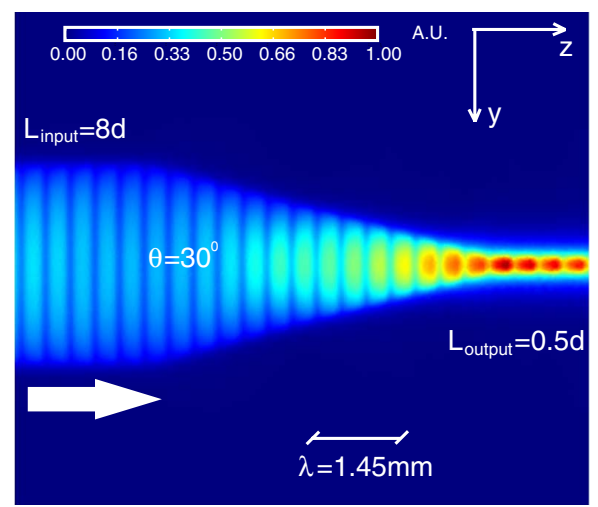

Fig. 3. (Color online) Poynting vector field (modulus) distribution in a $y z$-horizontal plane above $(x=0.5 d)$ the tapered TWG. The lateral width is changed gradually from $L_{\text {in }}=8 d$ to $L_{\text {out }}=$ $0.5 d$ with an angle $\theta=30^{\circ}$. The rest of the parameters are as in previous figures. The vector field is evaluated at $\lambda=1.45 \mathrm{~mm}$ and is in arbitrary units.

it is expected that the change in $L$ does not increase the scattering and reflection losses of the surface EM mode when it propagates along the tapered waveguide. As shown in Fig. 3, this is indeed the case in our structure. The device performance is analyzed at an operating wavelength of $1.45 \mathrm{~mm}$, where the lateral width of the input waveguide is $L=8 d$ and that of the output one is $L=0.5 d$, resulting in an aperture angle of $\theta=30^{\circ}$. By analyzing the Poynting vector both at the input and output sides of the device and on the boundaries of the 3D simulation region, the reflection loss is calculated to be less than $7 \%$ whereas the scattering loss is $3 \%$. Therefore, if we ignore ohmic losses, more than $90 \%$ of the input power is channeled through the narrow TWG.

In summary, we have presented a new type of terrahertz waveguides based on the concept of spoof surface plasmons. These waveguides possess large insensitivity to the lateral size and their propagation lengths are long-enough to think of building-up photonic devices made of them. We have illustrated the potentiality of these waveguides by presenting a tapered waveguide in which the energy carried out by a very wide waveguide is efficiently transferred to a narrow one at a very deepsubwavelength scale.

Work supported by the Spanish Ministry of Science and Innovation (MICINN) by grants MAT2008-06609-C02 and CSD2007-046-NanoLight.es. D. M.-C. acknowledges
Formacion del Personal Universitario (FPU) grant AP2007-00891 from the Spanish Ministry of Education.

\section{References}

1. B. Ferguson and X.-C. Zhang, Nat. Mater. 1, 26 (2002).

2. M. Tonouchi, Nat. Photon. 1, 97 (2007).

3. S. Withington, Phil. Trans. R. Soc. A 362, 395 (2004).

4. P. H. Siegel, IEEE Trans. Microwave Theory Tech. 52, 2438 (2004).

5. A. Huber, F. Keilmann, J. Wittborn, J. Aizpurua, and R. Hillenbrand, Nano Lett. 8, 3766 (2008).

6. H. Hübers, Nat. Photon. 4, 503 (2010).

7. J. Federici and L. Moeller, J. Appl. Phys. 107, 111101 (2010).

8. W. Huang, Y. Zhang, and B. Li, Opt. Express 16, 1600 (2008).

9. T.-I. Jeon and D. Grischkowsky, Appl. Phys. Lett. 88, 061113 (2006).

10. K. Wang and D. M. Mittleman, Nature 432, 376 (2004).

11. J. B. Pendry, L. Martin-Moreno, and F. J. Garcia-Vidal, Science 305, 847 (2004).

12. F. J. Garcia-Vidal, L. Martin-Moreno, and J. B. Pendry, J. Opt. A 7, S97 (2005).

13. S. Maier, S. R. Andrews, L. Martin-Moreno, and F. J. Garcia-Vidal, Phys. Rev. Lett. 97, 176805 (2006).

14. A. I. Fernandez-Dominguez, E. Moreno, L. Martin-Moreno, and F. J. Garcia-Vidal, Opt. Lett. 34, 2063 (2009).

15. Z. Gao, X. Zhang, and L. Shen, J. Appl. Phys. 108, 113104 (2010).

16. D. Martin-Cano, M. Nesterov, A. Fernandez-Dominguez, F. Garcia-Vidal, L. Martin-Moreno, and E. Moreno, Opt. Express 18, 754 (2010).

17. M. Nesterov, D. Martin-Cano, A. Fernandez-Dominguez, E. Moreno, L. Martin-Moreno, and F. Garcia-Vidal, Opt. Lett. 35, 423 (2010).

18. W. Zhao, O. Eldaiki, R. Yang, and Z. Lu, Opt. Express 18, 21498 (2010).

19. E. M. G. Brock, E. Hendry, and A. P. Hibbins, Appl. Phys. Lett. 99, 051108 (2011).

20. D. Sievenpiper, in Metamaterials (John Wiley \& Sons, Inc., 2006), pp. 285-311.

21. M. Lockyear, A. Hibbins, and J. Sambles, Phys. Rev. Lett. 102, 73901(2009).

22. J. D. Jackson, Classical Electrodynamics (John Wiley \& Sons, 1999).

23. M. A. Ordal, L. L. Long, R. J. Bell, S. E. Bell, R. R. Bell, R. W. Alexander, and C. A. Ward, Appl. Opt. 22, 1099 (1983).

24. M. Seo, H. Park, S. Koo, D. Park, J. Kang, O. Suwal, S. Choi, P. Planken, G. Park, N. Park, Q. H. Park, and D. S. Kim, Nat. Photon. 3, 152 (2009).

25. H. Raether, Surface Plasmons (Springer-Verlag, 1988).

26. E. Rajo-Iglesias, O. Quevedo-Teruel, and L. Inclan-Sanchez, IEEE Trans Antennas Propag. 57, 3852 (2009). 